\title{
Perfil parasitológico de Oreochromis niloticus y su relación con la calidad del agua en granjas acuícolas Tierra Caliente de Guerrero
}

Parasitological profile of Oreochromis niloticus and its relationship with water quality in Tierra Caliente de Guerrero aquaculture farms

Escobar Sarabia Ludybed ${ }^{1}$, Diana Pérez de Jesús ${ }^{\circledR}{ }^{\bowtie}$, Maribel Ramírez Orozco ${ }^{1}$ y Jesús Sánchez Espinoza ${ }^{1}$

${ }^{1}$ Academia de Biología, Instituto Tecnológico de Cd. Altamirano, Av. Pungarabato Pte. S/N Col. Morelos, Cd. Altamirano, Guerrero, México. CP. 40660. Tel y Fax: (767) 6721213

${ }^{凶}$ Autor para correspondencia: diana_jesus@hotmail.com

Recibido: $15 / 09 / 2018$

Aceptado: 15/11/2018

\section{RESUMEN}

La investigación se realizó en la región Tierra Caliente del Estado de Guerrero entre los meses de Enero a Octubre del año 2018. El objetivo fue determinar el perfil parasitológico de Oreochromis niloticus y su relación con la calidad del agua en granjas acuícolas de dicha Región Calentana ( representada por nueve municipios), ya que representa la zona de producción acuícola más grande y su principal fuente de abastecimiento de agua es el Rio Balsas y rio Cutzamala siendo uno de los ríos más contaminados del estado y país; por lo tanto utilizar sus aguas sin tratamientos de descontaminación para la producción de alimentos, representa un enorme riesgo a la salud humana. Para el estudio se muestrearon un promedio de siete granjas encontradas en las localidades de cada municipio de la región y se analizaron un total de 70 tilapias por municipio, teniendo un total 630 organismos, utilizando las técnicas de Disección, Raspado de piel y Coproparasitologicas, para la detección de parásitos adultos y huevos. Se encontraron 14 géneros de parásitos de los cuales cuatro son específicos de peces y diez de otros vertebrados, los géneros Phagobranchium sp y Trichodina $s p$ presentaron mayor prevalencia con un $34.44 \%$ y $32.22 \%$ respectivamente, siendo los de menor prevalencia Ascaris sp, Enterobious sp, Heterakis sp y Paragonimus sp con $14.11 \%$ cada género. Se determinó que los parámetros indicadores de calidad de agua en los cultivos de tilapia en región Calentana, no influye sobre la presencia de parásitos ya que no se encontró relación significativa entre dichas variables según el Coeficiente de Correlación de Pearson, ya que el p-valor es mayor que 0.05 , por lo tanto la presencia de parásitos en los cultivos de tilapias en región se le atribuye a la contaminación microbiológica de la fuente de abastecimiento de agua.

Palabras claves: Contaminación Parasitológica, Oreochromis niloticus, Epidemiología, prevalencia parasitaria.

\begin{abstract}
The investigation was carried out in the Tierra Caliente region of the State of Guerrero between the months of January to October of the year 2018. The objective was to determine the parasitological profile of Oreochromis niloticus and its relation with the water quality in aquaculture farms of said Calentana Region ( represented by nine municipalities), since it represents the largest aquaculture production area and its main source of water supply is the Balsas River and Cutzamala River being


one of the most polluted rivers in the state and country; therefore, using its waters without decontamination treatments for the production of food represents a huge risk to human health. For the study an average of seven farms found in the localities of each municipality of the region were sampled and a total of 70 tilapia were analyzed by municipality, with a total of 630 organisms, using the Dissection, Skin Scraping and Coproparasitological techniques, to the detection of adult parasites and eggs. We found 14 genera of parasites of which four are fish specific and ten from other vertebrates, the genera Phagobranchium $s p$ and Trichodina $s p$ had a higher prevalence with $34.44 \%$ and $32.22 \%$ respectively, being the least prevalent Ascaris sp, Enterobious sp, Heterakis $s p$ and Paragonimus $s p$ with $14.11 \%$ each gender. It was determined that the water quality indicator parameters in the tilapia cultures in the Calentana region do not influence the presence of parasites since no significant relationship was found between these variables according to the Pearson Correlation Coefficient, since the p-value is greater than 0.05 , therefore the presence of parasites in tilapia crops in the region is attributed to the microbiological contamination of the water supply source.

Keywords: Parasitological contamination, Oreochromis niloticus, Epidemiology, parasitic prevalence.

\section{INTRODUCCIÓN}

En el cultivo de peces la calidad del agua representa un aspecto fundamental, al permitir manejar altas densidades poblacionales, rápido crecimiento y buena calidad de la carne de los peces cultivados para consumo humano, por lo tanto, una mala calidad del agua representa un riesgo de gran magnitud para la salud de los peces que se cultivan, así como para las personas que lo consumen, además la contaminación que se presenta en aguas continentales, pueden afectar a los peces en cultivo al incrementar la susceptibilidad a enfermedades, entre las que se encuentran, micosis, bacteriosis y parasitosis (Bautista Covarrubias \& RuizVelazco 2011). La presencia de parásitos en peces de consumo humano es muy frecuente y tiene diversas consecuencias, relacionadas con aspectos económicos y sanitarios, mayormente cuando se producen bajo condiciones de mala calidad de agua (PereiraBueno \& Ferre Peréz 1997).

En la región de Tierra Caliente la acuicultura se realiza principalmente utilizando agua de río y se sabe que la mayoría de ellos presentan contaminación, por lo tanto la calidad del agua es mala con respecto a parámetros físicos químicos y biológicos. En el caso específico del Rio Balsas y Cutzamala donde se toma el agua para los cultivos de tilapia, sus aguas presentaron mala calidad según los últimos informes de calidad de agua realizados (Aburn University, 2001). Además Romero-Monge \& Romero-Rivera en el año 2012 encontraron en Atiocoyo coliformes totales y fecales en el agua de los estanques y el cuerpo de las tilapias por encima de los límites permisibles, por lo tanto se consideró necesario realizar más estudios en el área epidemiológica y principalmente en la parasitología de los cultivos de tilapia. Los parásitos han sido catalogados de gran importancia económica por los efectos negativos que produce en peces de cultivos dulceacuícolas (Arguedas, 2010). Entre los parásitos que se pueden encontrar en los peces producidos en acuicultura están los nemátodos (Anisakis sp., Pseudoterranova sp., Eustrongylides sp. y Gnathostoma sp.), los cestodos o solitarias (Diphyllobothrium 
sp.) y los tremátodos (Chlonorchis sinensis, Opisthorchis sp., Heterophyes sp., Metagonimus sp., Nanophyetes salmonicola y Paragonimus sp.) (Astilapia 2009).

Según Cantor (2007), la tilapia Oreochromis niloticus es un pez teleósteo, del orden Perciforme perteneciente a la familia Cichlidae. Originario de África. En las últimas décadas ha sido introducido prácticamente a todas las regiones del planeta que le permitan su desarrollo (VegaVillasante 2009). En la producción de peces de agua dulce siguen predominado las carpas (71,9 \%, 24,2 millones de toneladas, en 2010). La producción de tilapia tiene una amplia distribución; el $72 \%$ se cría en Asia (sobre todo en China y el sudeste asiático), el $19 \%$ en África y $9 \%$ en América. (FAO, 2012).La tilapia ocupa un lugar importante en la producción de las especies de peces, siendo China el principal productor seguido de Egipto, Tailandia e Indonesia. (FAO, 2012).La Organización Mundial de Sanidad Animal (OIE), ha desempeñado su cometido bajo la autoridad y el control de una asamblea mundial, compuesta de delegados que designan los gobiernos de los países miembros. Garantizando la transparencia de la situación zoosanitaria en el mundo. Recopilando, analizando y difundiendo la información científica veterinaria.

\section{METODOLOGÍA}

Para determinar el tamaño de la muestra a nivel de granjas, se utilizó el software Win Episcope, el cual es empleado ampliamente en el diseño de investigaciones en el área de la epidemiologia cuántica, la realización de estudios epidemiológicos y específicamente la selección del tamaño de la muestra, esta metodología de muestreo fue diseñada para detectar si más de un numero especifico o porcentaje mayor que cero de tilapias están contaminadas por organismos patógenos (Frankena \& Goelema 1990).

Mediante la siguiente ecuación se determinó el tamaño de la muestra:

$\mathrm{n}=\{1-(1-\mathrm{a})\}\{\mathrm{N}-(\mathrm{D}-1) / 2\}$

Dónde:

$\mathrm{n}=$ Tamaño de la muestra .

$\mathrm{N}=$ Tamaño de la población (95 granjas).

$\mathrm{D}=$ Número de animales enfermos (25) (mortalidad).

$\mathrm{a}=$ Nivel de confianza (95\%).

$\mathrm{p}=$ Prevalencia estimada (se estima mediante los datos del número de estanques y la cantidad de tilapias muertas por estanque cundo estos datos son procesados en el software).

Al realizar el análisis mediante el software Win Episcope se tiene que:

$\mathrm{n}=$ Tamaño de la muestra: 10 granjas.

$\mathrm{N}$ = Tamaño de la población: 63 granjas.

$\mathrm{D}=$ Número de tilapias enfermas (mortalidad): 25.

$\mathrm{a}=$ Nivel de confianza: $95 \%$.

$\mathrm{p}=$ Prevalencia estimada: $30 \%$.

Las granjas cuentan con un promedio de 5 estanques, bajo los mismos tratamientos de mantenimiento del cultivo, por tal motivo se muestreó un solo estanque por cada granja. Para seleccionar el número de tilapias que se examinaron por estanque, se utilizó el número de siembra por estanque, en la región es de 10,000 tilapias aproximadamente y la prevalencia obtenida con el software Win Episcope que es de $30 \%$, utilizando el tamaño de población, tamaño de la muestra necesaria para obtener el porcentaje de prevalencia como es: $2 \%, 5 \%, 10 \%, 30 \%, 40 \%, 50 \%$, cabe mencionar que esta es utilizada por la OIE (Organización Mundial de Sanidad Animal) para el muestreo acuícola. 
Tabla 1. Selección del tamaño de muestra, basado en el porcentaje de prevalencia de un patógeno en una población determinada.

\begin{tabular}{|c|c|c|c|c|c|c|c|}
\hline \multirow{2}{*}{$\begin{array}{c}\text { Tamaño de } \\
\text { la poblacion }\end{array}$} & \multicolumn{5}{|c|}{ Tamaño de la muestra necesaria para obtener el porcentaje de prevalencia*z } \\
\cline { 2 - 9 } & $2 \%$ & $5 \%$ & $10 \%$ & $20 \%$ & $30 \%$ & $40 \%$ & $50 \%$ \\
\hline 50 & 50 & 35 & 20 & 10 & 7 & 5 & 2 \\
\hline 100 & 75 & 45 & 23 & 11 & 9 & 7 & 6 \\
\hline 250 & 110 & 50 & 25 & 10 & 9 & 8 & 7 \\
\hline 500 & 130 & 55 & 26 & 10 & 9 & 8 & 7 \\
\hline 1,000 & 140 & 55 & 27 & 10 & 9 & 9 & 8 \\
\hline 1,500 & 140 & 55 & 27 & 10 & 9 & 9 & 8 \\
\hline 2,000 & 145 & 60 & 27 & 10 & 9 & 9 & 8 \\
\hline 4,000 & 145 & 60 & 27 & 10 & 9 & 9 & 8 \\
\hline 10,000 & 145 & 60 & 27 & 10 & 9 & 9 & 8 \\
\hline$>10,000$ & 150 & 60 & 30 & 10 & 9 & 9 & 8 \\
\hline
\end{tabular}

Tomado de (Morales \& Cuéllar-Anjel 2008).

\section{Metodología de campo}

Se examinaron 7 tilapias de talla comercial de cada uno de los 7 estanques que se muestrearon, el total de tilapias examinadas fue de 630 individuos y se recogieron los datos de los parámetros indicadores de calidad de agua para cada estanque. En el estanque de cultivo seleccionado, de forma directa y puntual se tomaron los datos de los parámetros indicadores de calidad de agua al momento que se tomó la muestra de tilapias (pH, Turbidez, Temperatura y Oxígeno disuelto), para ello se utilizó un equipo multiparámetro con el que se obtuvieron datos de temperatura y $\mathrm{pH}$, el oxígeno disueltos se midió con un oxigenómetro, los datos de turbidez se tomaron con un disco secchi. Las 10 tilapias fueron capturadas al azar en el estanque seleccionado de cada granja mediante la utilización de una atarraya, luego se depositaron en una bolsa plástica con suficiente agua y oxígeno, posteriormente fueron transportadas hasta el Laboratorio de Zoología del Instituto Tecnológico de $\mathrm{Cd}$. Altamirano, donde fueron aclimatadas $\mathrm{y}$ trasladadas a peceras para mantenerlas con vida previo al examen parasitológico, para evitar el deterioro de las muestras. La identificación de la fauna parasitaria se realizó aplicando la siguiente metodología, según Cordero del Campillo et al. (1999), Soulsby (1987) y FAO (2013):

\section{Necropsia de los peces para detectar parásitos}

La secuencia del examen es según (FAO 2013). Los peces a examinados fueron retirados de la pecera y se colocaron en una bandeja de disección donde se sacrificaron por medio del corte de las branquias. Enseguida se realizó un raspado de piel en la región caudal y los costados del pez, posteriormente se colocaron en un portaobjeto con dos gotas de agua y se examinó utilizando un microscopio compuesto de campo claro en busca de protozoarios y monogeneos pequeños. Luego se examinó la superficie exterior, incluyendo las cavidades bucales y operculares con un microscopio compuesto estereoscopio. Posteriormente se tomaron las branquias antes retiradas para examinarlas individualmente con un microscopio 
compuesto estereoscopio. Enseguida se extrajo el intestino y se examinó el exterior, luego se cortó en secciones, se abrió cada sección desde la parte posterior hasta la anterior y se observó en busca de parásitos adultos, finalmente se extrajo el contenido intestinal para posteriormente realizar el estudio coproparasitológico. Su musculatura se examinó preparando porciones aplastadas cortadas con un bisturí quirúrgico para buscar quistes de parásitos, se realizó la prueba a trasluz utilizando un microscopio compuesto estereoscopio y un microscopio compuesto de campo claro.

\section{Estudio coprológico para la identificación de huevos de parásitos}

Para realizar el estudio coprológico de huevos de parásitos, se elaboró una solución sobre saturada de azúcar, para identificar parásitos, ya sean platelmintos o nematelmintos. Se aplicó el análisis de flotación en serie y el análisis de sedimentación; por ser las técnicas más utilizadas en nuestro medio, además son considerados los más eficaces y de bajo costos. Para preparación de la solución sobresaturada de azúcar, se colocó en un recipiente de aluminio se depositaron $1000 \mathrm{cc}$ de agua corriente, luego se agregó 1280 g de azúcar y se calentó en una cocina a temperatura moderada, mezclando la solución con un agitador de vidrio, hasta que la solución se homogenizo completamente evitando que hierva, posteriormente se dejó enfriar a temperatura ambiente y se agregó 10 $\mathrm{ml}$ de fenol para evitar el crecimiento de hongos y otros microorganismos, después de preparada la solución se almacenó a temperatura ambiente en botellas plásticas, para su posterior utilización. Para su diagnóstico coproparasitológico Se llevó a cabo en tres fases: 1. Examen directo al fresco consistio en colocar dos gotas de agua en un porta objeto y se tomó con la punta de un palillo una pequeña porción de heces (1 gramo) extraídas del intestino de los peces muestreados. Luego se procedió a homogenizar la muestra con la ayuda de un palillo de dientes, se colocó un cubre objeto y se examinó al microscopio compuesto de campo claro con el objetivo 10X y 40X. Con la ayuda de las claves e ilustraciones de Soulsby (1987), Cordero del Campillo et al. (1999), Kinkelin et al. (1991), Rebuffo \& Almada (2008), CECOPESCA (2012) y apoyo en la web, se identificaron los huevos y larvas de parásitos. Para el método de flotación en serie con solución sobresaturada de azúcar, en un mortero se colocó aproximadamente cuatro gramos de heces y se agregaron $15 \mathrm{ml}$ de la solución sobresaturada de azúcar, se homogenizo suavemente con el pistilo del mortero hasta lograr una suspensión adecuada. Luego se tamizó con un colador corriente, el filtrado se depositó en un beaker de $50 \mathrm{ml}$. Posteriormente el filtrado se colocó en un tubo de ensayo de $10 \mathrm{ml}$, tratando de que el menisco quedara convexo. Enseguida se colocó un cubre objeto sobre el menisco y se dejó en esa posición durante diez minutos. Finalmente se procedió a transferir el cubre objeto a una lámina porta objeto, para llevarlo al microscopio compuesto de campo claro, observando uno de los extremos superiores del porta objeto para realizar la lectura en forma de zigzag y enfocando de ser posible hasta 100X para observar estructuras de los huevos. Con la ayuda de las claves e ilustraciones de Soulsby (1987), Cordero del Campillo et al. (1999), Kinkelin et al. (1991), Rebuffo \& Almada (2008), CECOPESCA (2012) y apoyo en la web, se identificaron los huevos y larvas de parásitos. Finamente para el método de análisis de sedimentación, este se realizada mediante la fase del método de flotación en serie con solución sobresaturada de azúcar, al contenido que quedo en el tubo de ensayo después de retirado el cubre objetos se le agregó cierta cantidad de agua para reducir la 
densidad y lograr que el sobrenadante se sedimentara. Luego con una pipeta, se tomó una porción del sedimento y se colocó en una lámina porta objeto y se llevó al microscopio compuesto de campo claro. Con la ayuda de las claves e ilustraciones de Soulsby (1987), Cordero del Campillo et al. (1999), Kinkelin et al. (1991), Rebuffo \& Almada (2008), CECOPESCA (2012) y apoyo en la web, se identificaron los huevos y larvas de parásitos. Los datos de los parásitos fueron incorporados en una tabla que contiene la información de la granja donde fue tomada la muestra y el órgano del pez donde se encontraron los parásitos.

Para analizar la ocurrencia de parásitos en los estanques, se realizó una tabla de frecuencias y el cálculo de la prevalencia parasitaria por estanque, que nos permitió conocer el porcentaje de organismos parasitados por una especie de parasito.

La prevalencia se calculó mediante la siguiente formula:

Dónde:

$\mathrm{P}=\%$ prevalencia

\begin{tabular}{|l|c|c|}
\hline \multirow{2}{*}{$\mathrm{P}=$} & $\mathrm{N}^{\mathrm{o}}$ hospederos infectados & \multirow{2}{*}{$\mathrm{X} 100$} \\
\cline { 2 - 3 } & $\mathrm{N}^{\mathrm{o}}$ hospederos examinados & \\
\hline
\end{tabular}

Finalmente con el objetivo de relacionar la calidad del agua con la presencia de

Parásitos, se verifico que los datos cumplan los supuestos de normalidad (prueba de Shapiro-Wilk) y homocedasticidad (prueba de Levene's), para luego, realizar un análisis de Coeficiente de Correlación Lineal de Pearson, mediante a siguiente ecuación:

$$
r_{x y}=\frac{N \sum X Y-\sum X \sum Y}{\sqrt{N \sum X^{2}\left(\sum X\right)^{2} \sqrt{ } N \sum Y^{2}\left(\sum Y\right)^{2}}}
$$

Para ello se utilizaron los programas estadísticos STATGRAPHICS Centurión y Past.

\section{RESULTADOS Y DISCUSIÓN}

Se examinaron 653 tilapias de las cuales 434 se encontraron infestadas por parásitos, esto representa el $48.6 \%$ del total de la muestra examinada, esto concuerda con lo encontrado por Contreras-Mata en el año 2007, quien investigó los agentes patógenos en estanques reproductores de tilapia. Examinó 473 muestras, de las cuales encontró el $41 \%$ con presencia de parásitos. Se encontró el parasito del género Gyrodactylus, el cual es uno de los parásitos más frecuentes a nivel de piel y branquias en tilapia, ya que en granjas acuícolas de región calentana ha sido encontrado en los estudios realizados por
Contreras-Mata en el año 2007 y AndasolSerrano et al. en el año 2014. Diphyllobothrium latum es un parásito propio de peces de agua dulce y salada, según Cordero del Campillo 1999 y Soulby 1982, en el presente estudio se detectó la presencia de huevos de este parásito en tilapias, en el Salvador se ha encontrado a Diphyllobothrium latum en estudios exploratorios pero no se tiene registro científico de este parasito en el país, que además de parasitar las tilapias también parasita al humano utilizándolo como huésped definitivo al igual que otros vertebrados piscícolas. Andasol-Serrano et al. en el año 2014, realizo un estudio de ectoparásitos en 
tilapias provenientes de laboratorios certificados por el Ministerio de Agricultura y Ganadería, ubicados en cinco departamentos de El Salvador, encontró alta prevalecía de las especies de ectoparásitos entre ellos Trichodina $s p$ con prevalencia de $91.67 \%$, lo que concuerda con lo encontrado en la presente investigación donde los ectoparásitos presentaron el mayor 53 porcentaje de prevalencia, donde Trichodina sp $\mathrm{y}$ phagobramchium presentaron la mayor prevalencia con un $22.22 \%$ y $24.44 \%$ respectivamente.

La clase que presento mayor número de individuos fue la clase nematoda con un total de ocho especies, mientras que la case Oligohymenophorea, Kinetofragminophorea y la clase Cestoda presentan el menor número con una especie respectivamente, según Verbel \& Avila (2008), las Nematodosis son las principales en enfermedades parasitarias que afectan a los peces. Ya que pueden infectarse al ingerir alimento o agua contaminada por parásitos, o porque el parásito penetre la piel de los peces al tener contacto directo (Cecopesca 2012). Según Soulsby (1987), los huevos de muchos parásitos pueden mantenerse viables durante largos periodos de tiempo, que van desde unos pocos minutos hasta dos semanas en aguas residuales urbanas, hasta más de un mes en aguas de rio y hasta 159 días en pastos e incluso resistir procesos de tratamiento de aguas residuales; lo anterior explica la presencia de parásitos que no son específicos de peces en los cultivos de tilapia en Atiocoyo, como lo son los generos Ancylostoma, Ascaris, Enterobious, Heterakis, Oesophagostomum, Paragonimus, Strongyloides, Taenia, Toxocara y Trichostrongylus, ya que para dichos cultivos se utiliza agua del rio sucio que según MARN (2011) (2012) presenta altos niveles de contaminación y los parásitos encontrados poseen huevos muy resistentes y les favorece la humedad, por lo que las tilapias por su hábito alimenticio podrían actuar según Cordero de Campillo et al. (1999) como vectores, trasladando los huevos a un huésped definitivo sin que el parasito haya logrado ninguna fase de su ciclo de vida en ellas. Romero-Monge y Romero-Rivera en el año 2012, recomiendan realizar un análisis de los parámetros indicadores de calidad del agua en los estanques, en atención a tal recomendación, se realizó el estudio parasitológico relacionando los parámetros indicadores de calidad del agua (Temperatura, oxígeno disuelto y turbidez) con la carga parasitaria de las tilapias y se determinó que no existe relación significativa entre los parámetros indicadores de calidad de agua y la presencia de parásitos, debido a que los productores realizan un control muy estricto de tales parámetros, por lo que no se encontró mayor variación entre granjas, pero no se tomó en cuenta la calidad microbiológica del agua que se incorpora a los estanques de cultivo, lo que probablemente si influya de manera directa sobre las patologías relacionadas al cultivo de tilapia. IrahetaNavas en el año 2007, desarrollo una investigación sobre endoparásitos en tilapias en la que indicó la ausencia de especies de parásitos de importancia para la salud humana en el intestino de las tilapias, contrario a dicha investigación, se encontraron géneros de parásitos de importancia para la salud humana en el intestino de las tilapias, como: Ascaris $s p$, Enterobious sp, Taenia sp, Paroginimus $s p$, Diphillobotrium latum; esto se debe a la calidad microbiología del agua que se utiliza en los estanques de cultivo sin pasar previamente por un sistema de filtrado, que según Barbón-Menjívar, Handal-Jiménez y Turish-Guardado en el año 2009, la calidad del agua en la cuenca del rio sucio, presento niveles de contaminación por encima de los límites establecidos por la Norma de Calidad de Agua para contacto humano de la OMS. 


\section{CONCLUSIONES}

De un total de 653 tilapias examinadas 413 resultaron parasitadas, lo que representa un $48.6 \%$ de peces infectados con parásitos externos a nivel de branquias y piel e internos a nivel de tracto digestivo. Se encontró un total de 14 géneros de parásitos de los cuales solamente cuatro géneros son parásitos propiamente de peces, los diez géneros restantes son comunes en otros vertebrados como bovino, porcinos, aves y humanos, aunque no se descarta que puedan parasitar peces. Se encontraron ocho géneros del Phylum Nematelminthes (Toxocara, Trichostrongylus, Strongyloides, Ancylostoma, Ascaris, Enterobius, Heterakis,Oesophagostomum), cuatro géneros del Phylum Platyhelminthes (Gyrodactylus, Diphyllobothrium, Paragonimus, Taenia), dos del Phylum Ciliophora (Trichodina, Phagobranchium) y no se encontró individuos del Phylum Arthropoda. En las diez granjas examinadas no se encontró variación en cuanto al número de parásitos encontrados a excepción de los géneros de Trichodina y Phagobranchium que presentaron el mayor número de individuos en los peces, debido al ciclo biológico de estos organismos ya que presenta una alta tasa reproductiva. De acuerdo con la prevalencia Trichodina y Phagobranchium fueron los géneros más representativos en el estudio con una prevalencia de $22.22 \%$ y $24.44 \%$ respectivamente a diferencia de Ascaris sp, Enterobious sp, Heterakis sp y Paragonimus $s p$ que presentaron menor prevalencia con un $1.11 \%$ cada una de las cuatro especies. Se determinó que parámetros indicadores de calidad del agua, Turbidez, Oxígeno Disuelto y Temperatura no influyen sobre la presencia de parásitos en los cultivos en la región Calentana de Guerrero, ya que no se encontró relación significativa según la prueba de Coeficiente de Correlación de Pearson entre dichas variables, por lo que la contaminación parasitológica se le atribuye a la calidad microbiológica del agua, ya que no se aplica ningún tipo de tratamiento antes de introducirla al estanque. El mayor número de parásitos fueron identificados a nivel de huevo en el tracto digestivo debido al hábito alimenticio de los peces que al alimentarse de plancton además de consumir el alimento incorporan simultáneamente a su tracto digestivo huevos de parásitos que están suspendidos en la columna de agua de debido a que la fuente de abastecimiento posee altos niveles de contaminación microbiológica, además, se encontraron solamente tres géneros de parásitos en su forma adultos a nivel de branquias y piel.

\section{LITERATURA CITADA}

Andasol-Serrano, I. G., D. A. EscobarLopez \& N. I. Montes-Rivera. 2014. Caracterización ectoparasitológica de alevines (Oreochromis niloticus).

Arguedas, D., G. Dolz, J. J. Romero, A. E. Jiménez \& D. León. 2010. Centrocestus formosanus

(Opisthorchiida: Heterophyidae) como causa de muerte de alevines de tilapia gris Oreochromis niloticus (Perciforme: Cichlidae) en el Pacífico seco de Costa Rica. Rev. Biol. Vol. $58 \quad$ (4): $1453-1465$. https://doi.org/10.15517/rbt.v58i4.5423

Astilapia (Asociación Sinalonoense de productores de tilapia, C.A.). 2009.

Barbón-Menjívar, S. M., A. Y. HandalJiménez \& S. M. Turish-Guardado. 2009.

Bautista-Covarrubias, J. C. \& J. M. RuizVelazco. 2011. Calidad de agua para el cultivo de Tilapia en tanques de geomembrana.

Barrera, A. R. E. \& C. E. Paz. 2006. Control de alevines de tilapia (Oreochromis niloticus) (perciforme: Cichlidae) usando guapote lagunero (Parachromis dovii) (perciforme: Cichlidae). 
Botello, J. E. 2013. MANUAL DE PRÁCTICAS DE PARASITOLOGÍA. Facultad de Medicina Veterinaria y Zootecnia. Universidad Autónoma del Estado de México.

Brenes, R., G. Hangen \& G. Duarte. 1984. REVISION DE PARAGONIMUS y PARAGONIMIASIS EN CENTROAMERICA y PANAMA. Rev. Med. Hosp. Na/. NIños Costa RIca 19 (2):87-106.

Cantor, A. F. 2007. Manual de producción de tilapia. Secretaría de Desarrollo Rural del Estado de Puebla. Puebla. México. Pp 97.

Cardona, Z. Edison A. 2005. La Coprologia como Técnica de Diagnóstico. Escuela de Medicina Veterinaria. Facultad de Ciencias Agrarias. Universidad de Antioquía.

CECOPESCA (Centro Técnico Nacional de Conservación de Productos de la Pesca y la Acuicultura, Ministerio de Agricultura, Alimentación y Medio Ambiente). 2012.

Chaguay, V. Y. J. 2004. Evaluacion del crecimiento, en etapa de precria de tilapia raja (Oreochromis spp), utilizando cinco niveles de proteínas en tanques abiertos.

Contreras-Mata, I. G. 2007. Determinación de Agentes Patógenos en estanques reproductores de Tilapias y los factores predisponentes.

Cordero del Campillo, M. \& F. A. Rojas Vásquez. 1999. Parasitología Veterinaria. Editorial. McGraw-Hill Interamericana. España. Pp 968.

Cuamba-Leal, G. 2008. Toxocara canis. Universidad Michoacana de San Nicolás de Hidalgo Facultad de Medicina Veterinaria y Zootecnia. Tesis para obtener el título de Médico Veterinario Zootecnista.
Dirección Nacional de Acuicultura. 2004. Cultivo de tilapia. Viceministerio de Pesquería.

Delgado, O. \& A. J. Rodríguez-Morales. 2009. Aspectos clínico-epidemiológicos de la toxocariasis: una enfermedad desatendida en Venezuela y América Latina. Boletín de malariología Y salud ambiental.

Fey, D. A. 2009. Parásitos branquiales de cuatro grupos genéticos de tilapias, cultivadas en las zonas Centro-Norte del Estado de Veracruz.

Foronda-Rodríguez, P. 2002. Estudio faunístico y sistemático de helmintos de aves canarias. UNIVERSIDAD DE LA LAGUNA. Dpto. de Parasitología, Ecología y Genética Facultad de Farmacia.

Frankena K. \& J. O. Goelema. 1990. Agricultural University, Deparment of Animal Husbandry. Wageningen. Holanda.

Guzmán, I. A. 2011. Cultivo de tilapia en México. (Tesis para optar al grado de médico veterinario zootecnista). Facultad de medicina veterinaria y zootecnia. Universidad Michoacana de San Nicolás de Hidalgo, México.

Hsien-Tsang, S. \& M. Quintanilla. 2008. Manual sobre "Reproducción y cultivo de tilapia". Centro de Desarrollo de la Pesca y la Acuicultura (CENDEPESCA). El Salvador. Pp 68. Iraheta-

Navas, E. J. 2007. Determinación de endoparásitos en las tilapias de los criaderos más importantes de Santa Ana. (Tesis de Licenciatura en Medicina Veterinaria). Facultad de Medicina Veterinaria y Zootecnia. Universidad Salvadoreña Alberto Masferrer.

Jiménez-Ramírez, J. A. . 2007. Principales enfermedades en peces de acuario. 
Universidad Michoacana de San Nicolás de Hidalgo Facultad De Medicina Veterinaria Y Zootecnia.

Kinkelin, P., C. Michel \& P. Ghittno. 1991. Tratado de las enfermedades de los peces.

Lorenzo-Manzanarez, J. L. 2011. Efecto de tres métodos de cocción sobre el contenido nutricional de la mojarra Tilapia (Oreochromis sp.). Ingeniería en alimentos. Universidad Del Papaloapan.MARN (Ministerio de Medio Ambiente y Recursos Naturales). 2012. Informe de la Calidad de Agua de los Ríos de El Salvador. Año 2011. Dirección General del Observatorio Ambiental Gerencia de Hidrología.

Morales, V. \& J. Cuéllar-Anjel. 2008. Guía Técnica - Patología e Inmunología de Camarones Penaeidos. Programa CYTED Red II-D Vannamei, Panamá, Rep. De Panamá. 270 pp.

Orta-Mira N., M. R. Guna Serrano. J. L. Pérez Sáenz \& C. Gimeno Cardona. 2007. DIAGNÓSTICO DE LAS TENIASIS INTESTINALES. Control de calidad SEIMC.
Pereira, A \& M. Pérez. 2001. Nematodosis intestinales. Parasitología. Facultad de Farmacia. Universidad de Santiago. Chile.

Pereira-Bueno, J. M. \& I. Ferre-Pérez. 1997. Parásitos del pescado. Universidad de León Departamento de Patología Animal: Sanidad Animal.

Rebuffo, N. \& M. Almada. 2008. Guía de platelmintos, Nemertinos y Ectoproctos. Provincia de Santa Fe.

Rodríguez, H., G. Polo \& G. Salazar. 1993. Fundamentos de acuicultura continental. Ministerio de Agricultura.

Romero-Monge, M. Y. \& M. H. Romero Rivera 2012. Determinación del perfil bacteriológico de Orechromis niloticus (Tilapia) fresca y su respectiva agua de estanque proveniente del Cantón Atiocoyo, Municipio de San Pablo Tacachico, La Libertad.

Verbel, J. O. \& R. B. Ávila. 2008. Parásitos en peces colombianos. Universidad de Cartagena. Facultad de Ciencias Farmacéuticas. Grupo de Química Ambiental y Computacional. Corporación Autónoma Regional del Canal del Dique (CARDEIQUE).

Copyright (c) 2018 Ludybed Escobar Sarabia, Diana Pérez de Jesús, Maribel Ramirez Orozco y Jesús Sánchez Espinoza

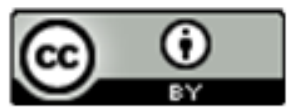

Este tex to está protegido por una licencia $\underline{\text { Creative Commons }} \underline{4.0}$

Usted es libre para Compartir — copiar y redistribuir el material en cualquier medio o formato-y Adaptar el documento — remezclar, transformar y crear a partir del material- para cualquier propósito, incluso para fines com erciales, siempre que cumpla la condición de:

Atribución: Usted debe dar crédito a la obra original de manera adecuada, proporcionar un enlace a la licencia, e indicar si se han realizado cambios. Puede hacerlo en cualquier forma razonable, pero no de forma tal que sugiera que tiene el apoyo del licenciante o lo recibe por el uso que hace de la obra. 